\title{
Laser measurements of the density shifts of resonance lines in antiprotonic helium atoms and stringent constraint on the antiproton charge and mass
}

\author{
H. A. Torii, ${ }^{*}$ R. S. Hayano, M. Hori, and T. Ishikawa \\ Department of Physics, University of Tokyo, 7-3-1 Hongo, Bunkyo-ku, Tokyo 113-0033, Japan \\ N. Morita and M. Kumakura \\ Institute for Molecular Science, Myodaiji, Okazaki 444-8585, Japan \\ I. Sugai and T. Yamazaki ${ }^{\dagger}$ \\ Institute of Particle and Nuclear Studies, High Energy Accelerator Research Organization, 3-2-1 Midori-cho, Tanashi, \\ Tokyo 188-8501, Japan \\ B. Ketzer, F. J. Hartmann, T. von Egidy, R. Pohl, and C. Maierl \\ Physik-Department, Technische Universität München, D-85747 Garching, Germany \\ D. Horváth \\ KFKI Research Institute for Particle and Nuclear Physics, H-1525 Budapest, Hungary \\ J. Eades and E. Widmann \\ CERN, CH-1211 Geneva 23, Switzerland
}

(Received 3 June 1998)

\begin{abstract}
We have performed laser spectroscopy of metastable antiprotonic helium atoms $\left(\bar{p} \mathrm{He}^{+}\right)$formed in helium media of $0.2-8.0$ bars at 5.8-6.3 $\mathrm{K}$ and have observed a density dependence of the resonance vacuum wavelengths for the known transitions $(n, l)=(39,35) \rightarrow(38,34)$ and $(37,34) \rightarrow(36,33)$. They showed linear redshifts of $0.61 \pm 0.01 \mathrm{GHz}$ and $0.22 \pm 0.02 \mathrm{GHz}$ per $1 \mathrm{~g} / \mathrm{l}$, respectively. With the shift parameters above, the transition vacuum wavelengths were extrapolated to zero-density limits, yielding $\lambda_{0}=597.2570 \pm 0.0003 \mathrm{~nm}$ and $\lambda_{0}=470.7220 \pm 0.0006 \mathrm{~nm}$, respectively. These values, with a 0.5 -ppm precision, were compared with the result of recent theoretical calculations on the energy of the Coulombic three-body system, including relativistic corrections and the Lamb shift. The agreement between our experimental values and the calculations has become as good as $2 \times 10^{-6}$. This excellent agreement in turn provides a precise value of the antiproton Rydberg constant that surpasses the currently known precision and sets a severe constraint on the antiproton charge $\left(-Q_{p}^{-}\right)$and the mass $\left(M_{p}^{-}\right)$that both $\left|Q_{p}-Q_{p}^{-}\right| / e$ and $\left|M_{p}-M_{p}^{-}\right| / M_{p}$ be less than $5 \times 10^{-7}$, when a more precisely known constraint on the charge-to-mass ratio is combined. Thus we have opened a possibility of determining fundamental constants of the antiproton. [S1050-2947(99)04501-1]
\end{abstract}

PACS number(s): 36.10.-k, 42.62.Fi, 32.70.Jz, 06.20.Jr

\section{INTRODUCTION}

Since the discovery of anomalous longevity of antiprotons in helium media [1] we have studied this peculiar phenomenon and proved that it is due to the formation of metastable antiprotonic helium atoms $\left(\bar{p} \mathrm{He}^{+}\right)[2-6]$ by performing laser-induced annihilation spectroscopy $[7,8]$. We have so far found 13 resonance lines and clarified the level structure, lifetime, and population of $\bar{p} \mathrm{He}^{+}$atoms [9-19]. The measured transition energies for all the lines discovered showed good agreement with theoretical predictions [7,20-24] (especially with that of the molecular-expansion variational cal-

\footnotetext{
*Present address: Institute of Physics, Komaba, University of Tokyo, 3-8-1 Komaba, Meguro-ku, Tokyo 153-8902, Japan.

${ }^{\dagger}$ Present address: Japan Society for the Promotion of Science, 5-3-1 Kojimachi, Chiyoda-ku, Tokyo 102-0083, Japan.

${ }^{\star}$ Present address: Department of Physics, University of Tokyo, 7-3-1 Hongo, Bunkyo-ku, Tokyo 113-0033, Japan.
}

culation by Korobov [23] together with relativistic corrections [24]). These theoretical calculations are based on a three-particle $\left(\bar{p} e^{-} \mathrm{He}^{2+}=\bar{p} \mathrm{He}^{+}\right)$model in which the $\bar{p} \mathrm{He}^{+}$ atom is tacitly assumed to be isolated and in vacuum. Experimentally $[8]$ these exotic atoms were produced by stopping $\bar{p}$ particles in $(0.5-1)$-bar helium gas, cooled down to $5-10 \mathrm{~K}$ in order to (a) freeze out impurity gases that otherwise would strongly quench the metastability [2,5,16-18] and (b) to obtain a high enough density to produce a small stopping volume. In such relatively dense media the $\bar{p} \mathrm{He}^{+}$ atom is subject to frequent collisions with surrounding normal helium atoms so that the assumption of in-vacuum isolation is by no means true. In fact, under this condition we have observed that certain metastable level lifetimes are shortened drastically with increasing density, as reported in a separate paper [19]. Further effects of collisions are to shift the resonance frequencies away from their original values and to broaden the linewidth. In this paper we will focus on the observed frequency shifts, the evaluation of which be- 


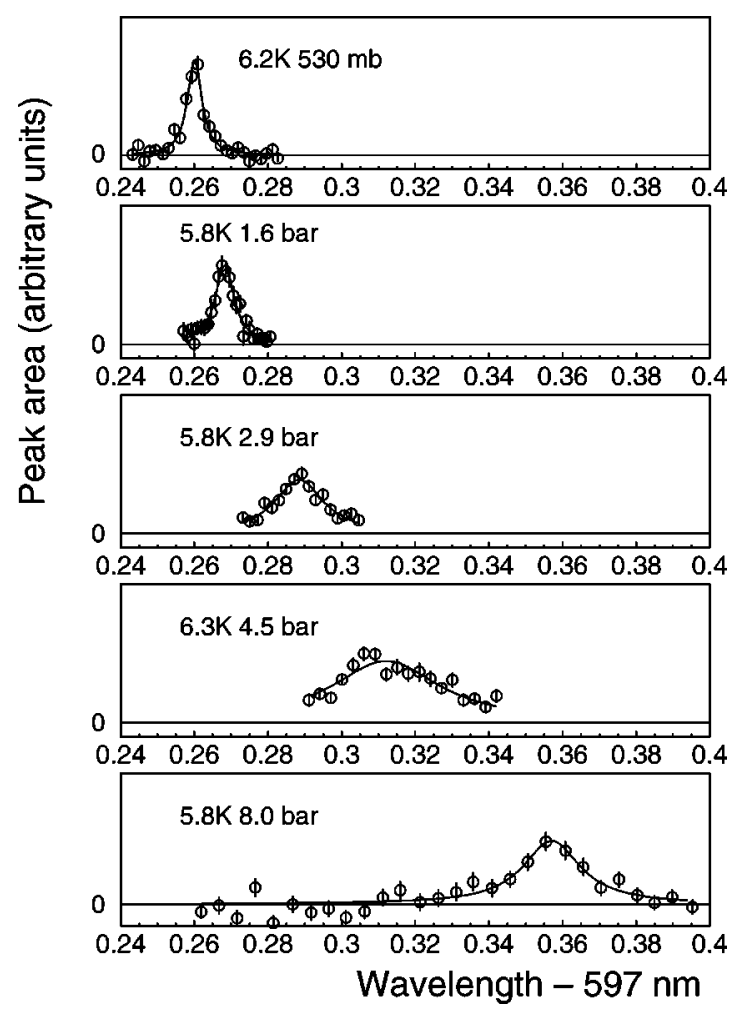

FIG. 1. Resonance profiles of the 597.26-nm line showing redshifts of the center with helium density. The linear scale for the $y$ axes is not the same for different target conditions.

came essential in determining precisely the unperturbed transition energies to be compared with the theoretical calculations.

\section{EXPERIMENTAL SETUP AND CONDITIONS}

The experimental apparatus was the same as that used in the former measurements [8] except for some modifications of the target chamber of the cryostat. For complete details on the experimental apparatus and technique, the reader is referred to Refs. [8] and [19]. Our method was to stimulate transitions between pairs of metastable $(\sim 1 \mu \mathrm{s})$ and shortlived ( $\sim 10 \mathrm{~ns})$ states and to observe resonant enhancement of antiproton annihilation, which follows the subsequent Auger transition to $\bar{p} \mathrm{He}^{2+}$ ions [7]. Since we irradiated each $\bar{p} \mathrm{He}^{+}$atom with its own laser pulse distributed spatially in the $\bar{p}$ stopping region with a large radial width of $1.5 \mathrm{~cm}$ [full width at half maximum (FWHM)], a high-power laser with a high repetition rate was required. We used a dye laser (Lambda Physik LPD3002E) pumped by a XeCl-excimer laser (LPX240i) at $\lambda=308 \mathrm{~nm}$. In contrast to our previous measurements, higher resolution of the laser frequency was required in view of possible small shifts. Thus the bandwidth (FWHM) was narrowed from the conventional $5 \mathrm{GHz}$ to typically $1 \mathrm{GHz}$ by using an intracavity étalon and aligning the lasers carefully; in particular we took care to suppress the amplified spontaneous emission. Under these conditions the narrowest achievable bandwidth was $1.0 \mathrm{GHz}$.

The frequency or, equivalently, vacuum wavelength was measured by a wavelength meter (Burleigh WA4500) that was calibrated with respect to standard iodine and tellurium
TABLE I. Summary of the shift measurements. Calibrated values for the central vacuum wavelengths of the two resonances at different target conditions (temperature, pressure, and corresponding density) are shown with relative errors that include both statistical and systematic errors for the relative wavelengths among different targets. In contrast to Fig. 2, the data points taken at similar conditions are averaged over in the table. The data written in italic were taken for the purpose of the absolute calibration of the wavelengths with respect to the standard reference lines and the errors given are the absolute errors instead.

\begin{tabular}{lccc}
\hline \hline$T(\mathrm{~K})$ & $p($ bars $)$ & $\rho(\mathrm{g} / \mathrm{l}) \pm \delta \rho$ & $\lambda(\mathrm{nm}) \pm \delta \lambda_{\text {rel/abs }}$ \\
\hline 6.2 & 0.17 & $1.38 \pm 0.03$ & $597.2582 \pm 0.0003$ \\
6.2 & 0.31 & $2.49 \pm 0.05$ & $597.2585 \pm 0.0003$ \\
15.2 & 1.00 & $3.17 \pm 0.03$ & $597.2598 \pm 0.0003$ \\
6.2 & 0.53 & $4.37 \pm 0.09$ & $597.2601 \pm 0.0003$ \\
5.8 & 0.54 & $4.78 \pm 0.10$ & $597.2606 \pm 0.0002$ \\
6.2 & 0.94 & $8.07 \pm 0.18$ & $597.2629 \pm 0.0004$ \\
5.8 & 1.56 & $16.1 \pm 0.6$ & $597.2681 \pm 0.0005$ \\
5.8 & 2.0 & $23.0 \pm 1.1$ & $597.2737 \pm 0.0006$ \\
5.8 & 2.9 & $45 \pm 5$ & $597.2885 \pm 0.0007$ \\
6.3 & 4.5 & $78 \pm 6$ & $597.3119 \pm 0.0022$ \\
5.8 & 8.0 & $127 \pm 2$ & $597.3570 \pm 0.0011$ \\
\hline 6.3 & 0.18 & $1.43 \pm 0.02$ & $470.7223 \pm 0.0003$ \\
5.8 & 0.50 & $4.37 \pm 0.09$ & $470.7229 \pm 0.0003$ \\
6.2 & 0.54 & $4.44 \pm 0.08$ & $470.7227 \pm 0.0003$ \\
5.8 & 0.59 & $5.21 \pm 0.11$ & $470.7224 \pm 0.0002$ \\
6.3 & 0.82 & $6.83 \pm 0.14$ & $470.7232 \pm 0.0006$ \\
6.3 & 1.17 & $10.1 \pm 0.2$ & $470.7237 \pm 0.0004$ \\
5.3 & 0.97 & $10.2 \pm 0.3$ & $470.7236 \pm 0.0017$ \\
6.0 & 1.18 & $11.0 \pm 0.3$ & $470.7239 \pm 0.0007$ \\
5.8 & 1.66 & $17.5 \pm 0.6$ & $470.7244 \pm 0.0013$ \\
5.8 & 2.5 & $32 \pm 2$ & $470.728 \pm 0.001$ \\
\hline \hline & & & \\
\hline
\end{tabular}

lines $[25,26]$. The error for the absolute values of the wavelength meter was specified as $600 \mathrm{MHz}$, but the relative error among different readings was found to be better from the reproducibility of the central wavelength of the $\bar{p} \mathrm{He}^{+}$resonance itself. The (systematic) error in relative frequency was thus $300 \mathrm{MHz}$.

We have observed density shifts of the resonance lines $(n, l)=(39,35) \rightarrow(38,34) \quad(597.26 \quad \mathrm{~nm}) \quad$ and $\quad(37,34)$ $\rightarrow(36,33)(470.72 \mathrm{~nm})$. These transitions lie at the ends of metastable radiative cascade series $v=n-l-1=3$ and 2, respectively. We measured resonance profiles by scanning the wavelength at different helium target conditions. Our data were taken at various laser powers. As expected, the laser power did not have any influence on the center of the resonance, though the width was increased for higher power on account of saturation broadening.

In a series of experiments in 1995, our target pressure was limited to a maximum of 1 bar by the strength of the quartz window of the target vessel. The temperature was kept at 6.2 $\mathrm{K}$, except for a few points taken at $15 \mathrm{~K}$. In the next year we improved the target system and took data at higher pressures up to 8 bars. We made use of the supercritical phase of helium, where the density increases with pressure continuously without a phase transition from gas to liquid. Thus we could achieve as high a density as that of liquid helium; 


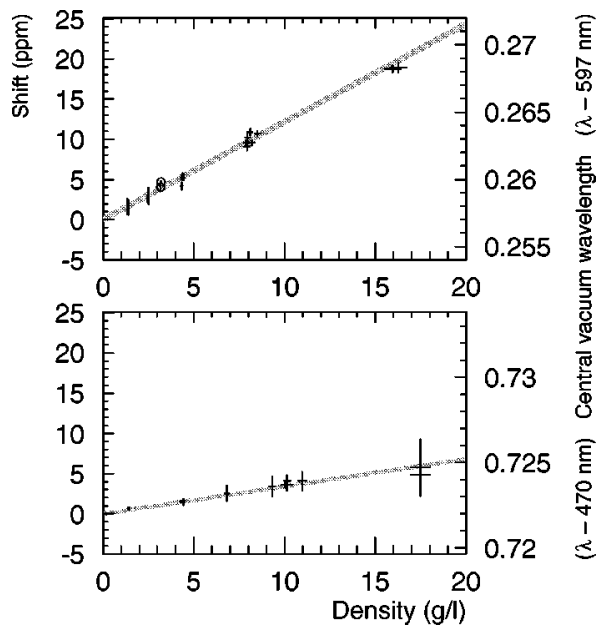

FIG. 2. Central vacuum wavelength plotted against the density of the helium target at relatively low densities. The encircled data taken at $15 \mathrm{~K}$ fall roughly on the linear line. The extrapolation to the zero-density limit gives the transition wavelengths of the $\bar{p} \mathrm{He}^{+}$ three-body system unperturbed by collisions. The line thickness covers the statistical error of the linear fit to the data.

helium of 8 bars at $5.8 \mathrm{~K}$ is denser than liquid helium ( $\rho$ $=125 \mathrm{~g} / \mathrm{l}$ at $4.2 \mathrm{~K}$ ) under atmospheric pressure. Many data were taken at $5.8 \mathrm{~K}$ with different target pressures, but we avoided the (3-5)-bar region, which is close to the critical point where the density changes drastically with slight pressure fluctuations. Instead, we took one data point at $6.3 \mathrm{~K}$ in order to do the measurement at a well-defined density.

\section{OBSERVED SHIFTS}

Figure 1 shows resonance profiles taken for the 597.26-nm line at different pressures ranging from $530 \mathrm{mb}$ to 8.0 bars at temperatures of $5.8-6.3 \mathrm{~K}$. The graphs plot the normalized count of the resonance peak area in the delayed annihilation time spectra [4-6] versus the calibrated vacuum wavelength. The center shifted toward longer wavelength and the width became broader for higher pressures. We determined the central vacuum wavelengths by fitting the profile data with a Lorentzian convoluted with a Gaussian of the laser bandwidth and plotted them against the target density. The target temperature and pressure were always monitored and recorded on the data tape and their calibrated values were converted into the density using a program [27] based on the cryogenic helium database. It was essential to use this database, especially at the supercritical phase where the ideal-gas assumption is totally broken.

The results at different target conditions are summarized in Table I and Fig. 2 (for lower density conditions), which show linear redshifts for both transitions studied. The data at $15 \mathrm{~K}$ fit very well on the line if plotted against density, but not against pressure. This means that the shift is proportional to the density $\rho$, with no significant dependence on the temperature. The vacuum wavelength $\lambda$ is thus expressed in the form

$$
\lambda=\lambda_{0}+s \rho,
$$
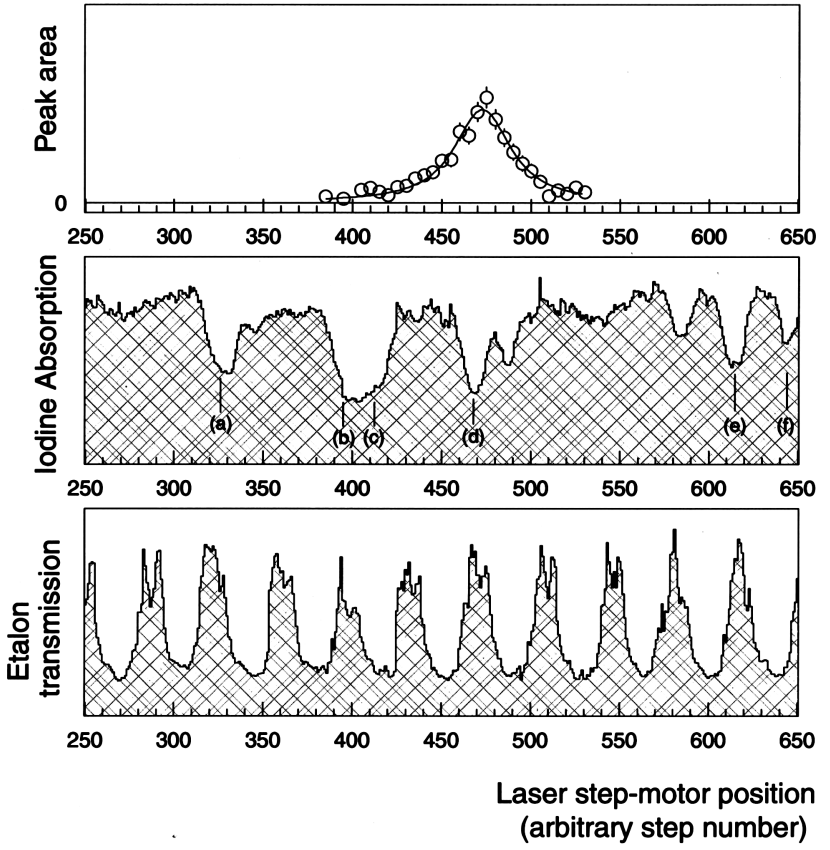

FIG. 3. A 597-nm resonance profile was taken with a target of $540 \mathrm{mb}$ at $5.8 \mathrm{~K}$, together with standard iodine-absorption lines in order to give an absolute wavelength calibration. $(a)-(f)$ correspond to reference lines listed in Table II. The central vacuum wavelength was determined to be $597.2606 \pm 0.0002 \mathrm{~nm}$.

where $\lambda_{0}$ is the extrapolated value at the zero-density limit.

In order to give a reliable calibration for the absolute wavelength values, one or two sets of resonance data for each transition in $\bar{p} \mathrm{He}^{+}$atoms were taken simultaneously with the standard atomic-absorption signals. Figure 3 shows a resonance profile of the 597-nm line taken at our common target condition of $540 \mathrm{mb}$ and $5.8 \mathrm{~K}$, together with the absorption and transmission signals from a standard iodine cell and a spectrum analyzer (an étalon) with a 3-GHz free spectral range. The central vacuum wavelength was obtained as $597.2606 \pm 0.0002 \mathrm{~nm}$ for this condition and this value was used as a standard for the absolute wavelength calibration in determining the values for other data (see Table I).

Figure 4 plots all the data at different target conditions, which show $0.61 \pm 0.01 \mathrm{GHz}$ and $0.22 \pm 0.02 \mathrm{GHz}$ redshifts per $1 \mathrm{~g} / \mathrm{l}$ for the 597-nm and 470-nm lines (see Table III). The shifts are linear within the experimental error, except for one data point at our maximum density of $127 \mathrm{~g} / \mathrm{l}=32 \mathrm{~mol} / \mathrm{l}$ for the 597-nm line. For that line the density has large errors at conditions near the critical point, due to the high sensitivity of the density on fluctuations of the temperature during the measurements. The fitting results gave the same shift slope within the error, regardless of whether or not these data were included in the fitting. In the case of the 470-nm transition, the measurement was limited to a maximum density $32 \mathrm{~g} / \mathrm{l}=8 \mathrm{~mol} / \mathrm{l}$ because the parent state $(n, l)=(37,34)$ was quenched by collisions at high density, reducing the height of the resonance peak until it disappeared as the density increased [19]. One interesting fact is that the 597-nm transition energy is more sensitive to collisions than the $470-\mathrm{nm}$ transition energy, while its lifetime shortening effect is much less marked [19]. These phenomena are not understood yet. 
TABLE II. List of reference $\mathrm{I}_{2}$ and $\mathrm{Te}_{2}$ absorption lines used for the wavelength calibration. The identification number of the lines and the wave number values are taken from Refs. [25,26] and are listed together with calculated corresponding vacuum wavelengths. $a-f$ correspond to the lines in Fig. 3 .

\begin{tabular}{|c|c|c|c|}
\hline Line & No. & $\begin{array}{l}\text { Wave number } \\
\qquad\left(\mathrm{cm}^{-1}\right)\end{array}$ & $\begin{array}{l}\text { Vacuum wavelength } \\
(\mathrm{nm})\end{array}$ \\
\hline \multicolumn{4}{|c|}{${ }^{128} \mathrm{I}_{2}$ reference lines ${ }^{\mathrm{a}}$} \\
\hline & 4509 & 16742.0419 & 597.29871 \\
\hline & 4510 & 16742.2272 & 597.29210 \\
\hline & 4511 & 16742.3125 & 597.28905 \\
\hline & 4512 & 16742.4869 & 597.28283 \\
\hline$a$ & 4513 & 16742.7148 & 597.27470 \\
\hline$b$ & 4514 & 16742.9009 & 597.26806 \\
\hline$c$ & 4515 & 16742.9484 & 597.26637 \\
\hline$d$ & 4516 & 16743.0984 & 597.26102 \\
\hline$e$ & 4517 & 16743.4965 & 597.24682 \\
\hline \multirow[t]{7}{*}{$f$} & 4518 & 16743.5754 & 597.24400 \\
\hline & 4519 & 16743.8554 & 597.23402 \\
\hline & 4520 & 16744.0762 & 597.22614 \\
\hline & \multicolumn{3}{|c|}{${ }^{130} \mathrm{Te}_{2}$ reference lines ${ }^{\mathrm{b}}$} \\
\hline & 59 & 21242.6542 & 470.75097 \\
\hline & 60 & 21243.4691 & 470.73291 \\
\hline & 61 & 21245.9655 & 470.67760 \\
\hline
\end{tabular}

${ }^{\text {a Reference [25]. }}$

${ }^{\mathrm{b}}$ Reference [26].

\section{TRANSITION WAVELENGTHS AT ZERO-DENSITY LIMITS}

The observation of the shifts has provided a more direct comparison between the experimental transition energies and the theoretical calculations. The wavelengths of the 13 known resonant transitions agree especially well with molecular-expansion variational calculations by Korobov [23]. The discrepancy found was generally about $50 \mathrm{ppm}$ for the "favored"' $(n, l) \rightarrow(n-1, l-1)$ transitions and about 100 ppm for the "unfavored"' $(n, l) \rightarrow(n+1, l-1)$ transitions. These discrepancies were further reduced to several ppm after taking into account the relativistic effect [24], as shown in Ref. [14]. In the figure therein, however, the experimental values compared were for our common condition of 0.5 bar at approximately $6 \mathrm{~K}$, while the theory calculated values for an isolated three-body atomic system, i.e., at the zero-density limit. Now that we know that density shifts occur for the 597- and 470-nm transitions, their extrapolated vacuum

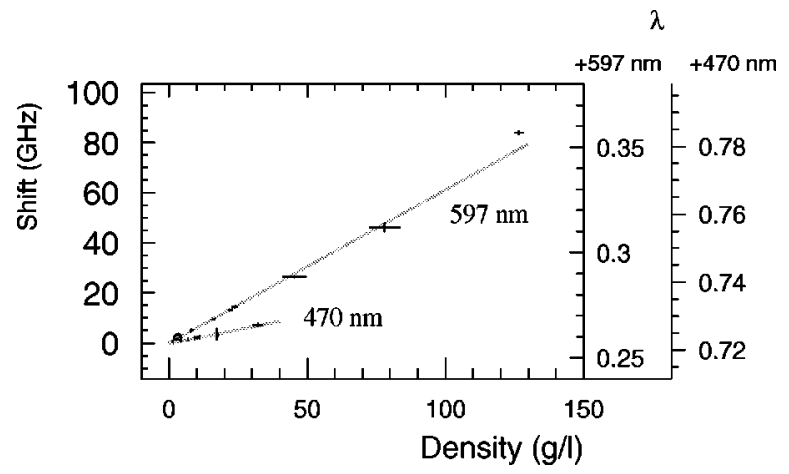

FIG. 4. Central vacuum wavelength plotted against the density of the target helium for the $(n, l)=(39,35) \rightarrow(38,34)(597-\mathrm{nm})$ and $(37,34) \rightarrow(36,33)(470-\mathrm{nm})$ resonance lines. No resonance was observed for the 470-nm line at density higher than $32 \mathrm{~g} / \mathrm{l}$ because of the quenched population of the parent state, which is no longer metastable.

wavelengths $\lambda_{0}$ are the ones that should be compared with the theoretical one.

We determined this value taking special care of the absolute wavelength calibration. Only the data at relatively low densities were used for the extrapolation. In this way we obtained $\lambda_{0}=597.2570 \pm 0.0003 \mathrm{~nm}$ and 470.7220 \pm 0.0006 $\mathrm{nm}$ for the two resonances. The errors include both statistical and systematic (calibration) errors and are smaller than the laser bandwidth. Thus the accuracies have improved by one order of magnitude compared to our earlier reports $[9,11]$ on the discovery of those resonances. These more recent values were compared with those of the relativistic calculation $\left(\lambda_{\mathrm{th}}\right)$ by Korobov [24] and small but significant discrepancies were revealed: $\left(\lambda_{\mathrm{th}}-\lambda_{0}\right) / \lambda_{0} \sim 8 \mathrm{ppm}$ for $597 \mathrm{~nm}$ and $6 \mathrm{ppm}$ for $470 \mathrm{~nm}$, as indicated by open circles in Fig. 5 .

Furthermore, recently Elander et al. [28-30] and Kino et al. [31,32] have come up with calculations including not only relativistic corrections but also the Lamb shift. Korobov has also revised his calculation in the meantime, using the atomic-expansion variational method instead [33]. All their values agree with each other, and with relativistic corrections and the Lamb shift taken into account, the discrepancies between theoretical and experimental values have become only a few ppm or less, as shown in Fig. 5. In particular, the latest values by Korobov and by Kino et al. agree with each other extremely well, in spite of the fact that they have used different computational methods with different basis sets. (Kino et al. use a coupled-rearrangement-channel variational method $[31,32]$.) Korobov now claims that his values for the nonrelativistic energies are accurate to $10^{-10}$ a.u. (corresponding to parts per $10^{9}$ order accuracies for transition en-

TABLE III. Extrapolated vacuum wavelengths $\lambda_{0}$ at the zero-density limit and shift parameters in various units. The larger error $\delta \lambda_{0}$ for the $470-\mathrm{nm}$ line than for the $597-\mathrm{nm}$ line is due to a larger error in the calibration of the absolute wavelength.

\begin{tabular}{lcccc}
\hline \hline & Wavelength & \multicolumn{2}{c}{ Density shift } \\
Transition & $\lambda_{0} \pm \delta \lambda_{0}$ & $\Delta \nu / \rho$ & $\Delta \nu / N$ & $(\Delta \lambda / \lambda) / \rho$ \\
$(n, l) \rightarrow\left(n^{\prime}, l^{\prime}\right)$ & $(\mathrm{nm})$ & {$[\mathrm{GHz} /(\mathrm{g} / \mathrm{l})]$} & $\left(10^{-21} \mathrm{GHz} / \mathrm{cm}^{-3}\right)$ & {$[\mathrm{ppm} /(\mathrm{g} / \mathrm{l})]$} \\
\hline$(39,35) \rightarrow(38,34)$ & $597.2570 \pm 0.0003$ & $-0.61 \pm 0.01$ & $-4.05 \pm 0.07$ & $1.22 \pm 0.02$ \\
$(37,34) \rightarrow(36,33)$ & $470.7220 \pm 0.0006$ & $-0.22 \pm 0.02$ & $-1.5 \pm 0.1$ & $0.34 \pm 0.03$ \\
\hline \hline
\end{tabular}



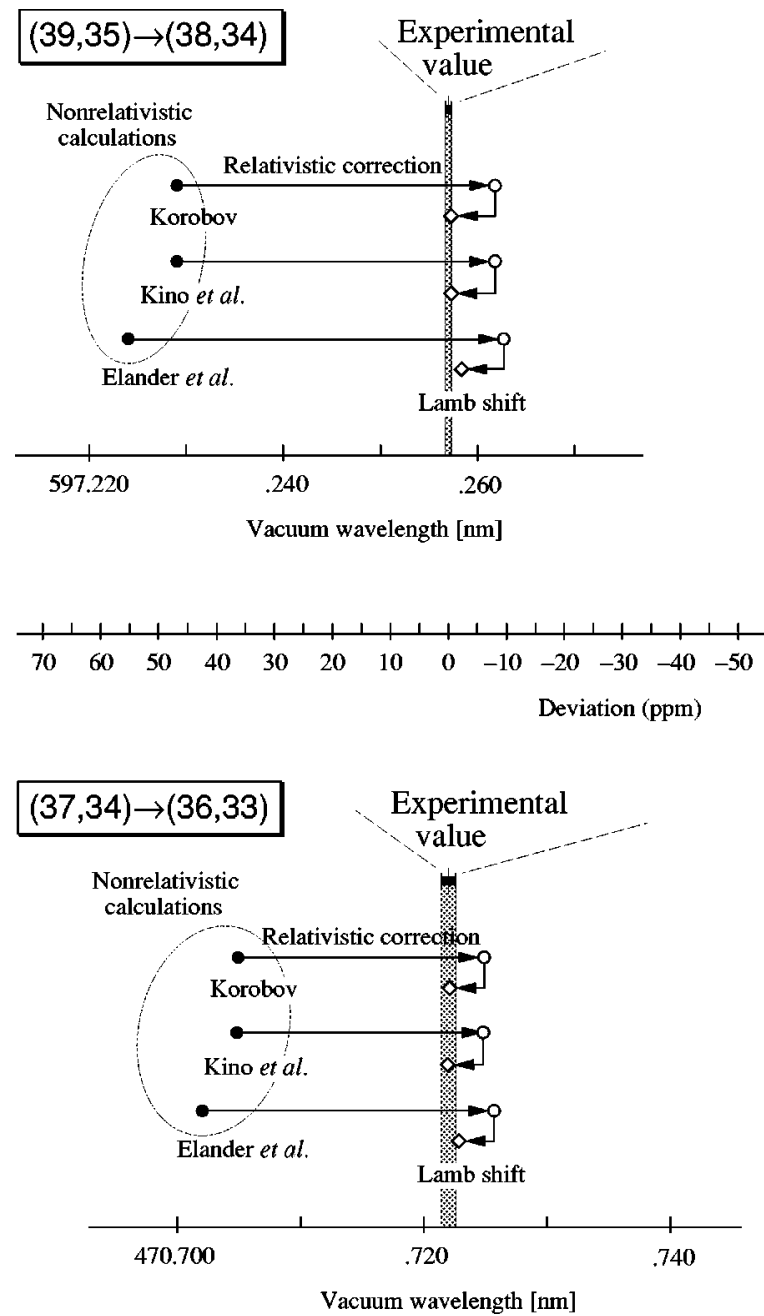

FIG. 5. Experimental values of the vacuum wavelengths for transitions $(39,35) \rightarrow(38,34)$ and $(37,34) \rightarrow(36,33)$ compared with recent theoretical values $[24,29,32,33]$, which agree within the precision of a few ppm when the relativistic corrections and the Lamb shift are taken into account. The so far known precision of the antiproton Rydberg constant (50 ppm) is comparable to the discrepancy in the figure between the nonrelativistic calculations and our experimental value, while the error of the present measurement of $0.5 \mathrm{ppm}$ (upper figure) is by far better than that. Note that the ppm scale applies to both transitions.

ergies) and that his relativistic values with the Lamb shift are precise within a computational uncertainty of $0.5 \mathrm{ppm}$ [33]. Also, his estimation shows that the contributions of higherorder relativistic and QED terms to the energies are at least one order of magnitude smaller than the Lamb shift [24], which justifies his values to the ppm accuracy. These theoreticians are currently working for even more accurate values and further results are anticipated.

\section{PRECISE DETERMINATION OF ANTIPROTON CHARGE AND MASS}

The excellent agreement between experiment and theory has opened a possibility of deducing fundamental constants of the antiproton by means of high-resolution spectroscopy of the $\bar{p} \mathrm{He}^{+}$atom. If we assume the CPT invariance between the properties of the proton and the antiproton, as the theoretical calculations do, the agreement is a signature of the excellence of theoretical treatments and calculation techniques of the Coulombic three-body system including QED corrections. On the other hand, if we take the calculation results for granted, this in turn gives a stringent test of the fundamental constants of the antiproton. In that sense, the current work provides the best test ground of the Rydberg constant $\left[R_{\infty}(\bar{p})\right]$, charge $\left(-Q_{\bar{p}}^{-}\right)$, and mass $\left(M_{\bar{p}}^{-}\right)$of the antiproton. From the agreement between the experimental and the theoretical results with their errors taken into consideration, we postulate the relation for the transition energies $(\Delta E)$ of the $\bar{p} \mathrm{He}^{+}$atoms

$$
\frac{\left|\Delta E_{\mathrm{th}}-\Delta E_{\text {expt }}\right|}{\Delta E}=\frac{\left|\lambda_{\mathrm{th}}-\lambda_{0}\right|}{\lambda_{0}}<2 \times 10^{-6}
$$

to deduce constraints on the fundamental constants.

The charge-to-mass ratio $Q_{\bar{p}}^{-} / M_{\bar{p}}$ of the antiproton is known with a high precision of $10^{-9}$ to be the same as that of the proton from a measurement of the cyclotron frequency in a trap experiment at Low Energy Antiproton Ring (LEAR), CERN by Gabrielse et al. [34]. This provides an extremely severe constraint as

$$
\left|\frac{\delta Q_{\bar{p}}}{e}-\frac{\delta M_{\bar{p}}}{M_{p}}\right|<1 \times 10^{-9},
$$

but does not mean that the charge and the mass were determined with that high precision. A poor constraint is known for $Q_{\bar{p}}$ (and $M_{\bar{p}}^{-}$) only to such a precision that $\left|Q_{p}-Q_{\bar{p}}^{-}\right| / e<2 \times 10^{-5}$ [35], from an x-ray measurement of antiprotonic atoms [36] that has determined the antiproton's Rydberg constant to a precision of $5 \times 10^{-5}$. The relation between the precision of the transition energy and that of the $\bar{p}$ charge and the mass is written in the following equation [35] if we neglect a small correction due to the finite mass of the heavy nucleus:

$$
\Delta E \propto R_{\infty}(\bar{p}) \equiv \frac{M_{\bar{p}}^{-} e^{2} Q_{\bar{p}}^{-2}}{2\left(4 \pi \varepsilon_{0}\right)^{2} \hbar^{2}} \propto Q_{\bar{p}}^{-3}\left(\frac{Q_{\bar{p}}^{-}}{M_{\bar{p}}^{-}}\right)^{-1} \propto M_{\bar{p}}{ }^{3}\left(\frac{Q_{\bar{p}}^{-}}{M_{\bar{p}}^{-}}\right)^{2}
$$

and thus

$$
\frac{\delta \Delta E}{\Delta E}=\frac{\delta R_{\infty}(\bar{p})}{R_{\infty}(\bar{p})}=3 \frac{\delta Q_{\bar{p}}^{-}}{e}=3 \frac{\delta M_{\bar{p}}^{-}}{M_{p}}
$$

since the value $Q_{p}^{-} / M_{p}^{-}$is precisely known [34].

In our case, the $\bar{p} \mathrm{He}^{+}$atom is a three-body system and the relation above may not be rigorously applied, but a similar equation should be used in order to set a new limit on the antiproton charge (and the mass) from the achieved precision of the $\bar{p} \mathrm{He}^{+}$transition energy. Very recently, Kino et al. have performed energy calculation [37] with $\bar{p}$ charge and mass that simultaneously deviate slightly from their original values (i.e., the values for the proton). The simultaneous deviation ensures the required condition that the charge-tomass ratio $Q_{\bar{p}}^{-} / M_{\bar{p}}$ be constant. Note that no deviation is added to the helium nucleus and the electron. Their results 
give $\mathrm{a}+(4.1-4.5)-\mathrm{ppm}$ shift of transition energies for the two resonances in question per +1 -ppm simultaneous deviation of the $\bar{p}$ charge and the mass:

$$
\frac{\delta \Delta E}{\Delta E}=-\frac{\delta \lambda_{0}}{\lambda_{0}}=f \frac{\delta Q_{\bar{p}}^{-}}{e} \quad\left(\frac{\delta Q_{\bar{p}}^{-}}{e}=\frac{\delta M_{\bar{p}}}{M_{p}}\right), \quad f \approx 4.1-4.5 .
$$

With this equation we obtain

$$
\frac{\delta Q_{\bar{p}}^{-}}{e}<5 \times 10^{-7}, \quad \frac{\delta M_{\bar{p}}^{-}}{M_{p}}<5 \times 10^{-7},
$$

which gives more than one order of magnitude better constraint on the antiproton charge and mass than the currently known value. Moreover, the experimental precision itself is as good as $\left|\delta \lambda_{0} / \lambda_{0}\right|=0.5 \mathrm{ppm}$ (for the 597-nm transition wavelength) and will be improved in the near future. So the potential limit is even narrower than the status quo as far as the experimental sector is concerned. So far, our data have been limited to the two transitions, but we are ready to extend these measurements to other transitions to test the theory. It would be most welcome that theoretical work in the near future advances to a precision below ppm, taking into account small effects including higher-order QED terms.

\section{CONCLUSION}

In summary, we have observed density shifts of the laser resonance lines of antiprotonic helium atoms in dense helium medium. The central frequency showed linear redshifts of $0.61 \pm 0.01 \mathrm{GHz}$ and $0.22 \pm 0.02 \mathrm{GHz}$ per $1 \mathrm{~g} / 1$ for the 597-nm and 470-nm transitions, respectively. The extrapolated values at zero-density limits were compared with recent theoretical calculations including relativistic and QED effects and showed agreement at precisions of $2 \mathrm{ppm}$. This already surpasses the currently known precision of the antiproton's Rydberg constant, giving the best constraint on the $\bar{p}$ charge and mass. Thus the high-precision spectroscopy of $\bar{p} \mathrm{He}^{+}$atoms has opened a possibility of measuring fundamental constants of the antiproton.

\section{ACKNOWLEDGMENTS}

We are deeply indebted to the LEAR and PS staff at CERN for their tireless dedication to providing us with the precious antiproton beam and to the CERN cryogenics group for invaluable help in modifying our cryostat. We are grateful to Dr. V.I. Korobov and Dr. Y. Kino for the stimulating discussions and for giving us their theoretical results prior to publication. The present work was supported by the Grantsin-Aid for Specially Promoted Research and for International Scientific Research of the Japanese Ministry of Education, Science and Culture, the German Bundesministerium für Forschung und Technologie, and the Hungarian National Science Foundation. H.A.T. acknowledges support of the Japan Society for the Promotion of Science (JSPS).
[1] M. Iwasaki, S.N. Nakamura, K. Shigaki, Y. Shimizu, H. Tamura, T. Ishikawa, R.S. Hayano, E. Takada, E. Widmann, H. Outa, M. Aoki, P. Kitching, and T. Yamazaki, Phys. Rev. Lett. 67, 1246 (1991).

[2] T. Yamazaki, E. Widmann, R.S. Hayano, M. Iwasaki, S.N. Nakamura, K. Shigaki, F.J. Hartmann, H. Daniel, T. von Egidy, P. Hofmann, Y.-S. Kim, and J. Eades, Nature (London) 361, 238 (1993).

[3] S.N. Nakamura, R.S. Hayano, M. Iwasaki, K. Shigaki, E. Widmann, T. Yamazaki, H. Daniel, T. von Egidy, F.J. Hartmann, P. Hofmann, Y.-S. Kim, and J. Eades, Phys. Rev. A 49, 4457 (1994).

[4] E. Widmann, I. Sugai, T. Yamazaki, R.S. Hayano, M. Iwasaki, S.N. Nakamura, H. Tamura, T.M. Ito, A. Kawachi, N. Nishida, W. Higemoto, Y. Ito, N. Morita, F.J. Hartmann, H. Daniel, T. von Egidy, W. Schmid, J. Hoffmann, and J. Eades, Phys. Rev. A 51, 2870 (1995).

[5] E. Widmann, I. Sugai, T. Yamazaki, R.S. Hayano, M. Iwasaki, S.N. Nakamura, H. Tamura, T.M. Ito, A. Kawachi, N. Nishida, W. Higemoto, Y. Ito, N. Morita, F.J. Hartmann, H. Daniel, T. von Egidy, W. Schmid, J. Hoffmann, and J. Eades, Phys. Rev. A 53, 3129 (1996).

[6] B. Ketzer, F.J. Hartmann, H. Daniel, T. von Egidy, A. Niestroj, S. Schmid, W. Schmid, T. Yamazaki, I. Sugai, K. Nakayoshi, R.S. Hayano, F.E. Maas, H.A. Torii, T. Ishikawa, H. Tamura, N. Morita, D. Horváth, J. Eades, and E. Widmann, Phys. Rev. A 53, 2108 (1996).
[7] N. Morita, K. Ohtsuki, and T. Yamazaki, Nucl. Instrum. Methods Phys. Res. A 330, 439 (1993).

[8] H.A. Torii, R.S. Hayano, F.E. Maas, N. Morita, M. Kumakura, T. Yamazaki, H. Masuda, I. Sugai, B. Ketzer, F.J. Hartmann, H. Daniel, T. von Egidy, W. Müller, W. Schmid, A. Niestroj, D. Horváth, J. Eades, and E. Widmann, Nucl. Instrum. Methods Phys. Res. A 396, 257 (1997).

[9] N. Morita, M. Kumakura, T. Yamazaki, E. Widmann, H. Masuda, I. Sugai, R.S. Hayano, F.E. Maas, H.A. Torii, F.J. Hartmann, H. Daniel, T. von Egidy, B. Ketzer, W. Müller, W. Schmid, D. Horváth, and J. Eades, Phys. Rev. Lett. 72, 1180 (1994).

[10] R.S. Hayano, F.E. Maas, H.A. Torii, N. Morita, M. Kumakura, T. Yamazaki, H. Masuda, I. Sugai, F.J. Hartmann, H. Daniel, T. von Egidy, B. Ketzer, W. Müller, W. Schmid, D. Horváth, J. Eades, and E. Widmann, Phys. Rev. Lett. 73, 1485 (1994); 73, 3181(E) (1994).

[11] F.E. Maas, R.S. Hayano, T. Ishikawa, H. Tamura, H.A. Torii, N. Morita, T. Yamazaki, I. Sugai, K. Nakayoshi, F.J. Hartmann, H. Daniel, T. von Egidy, B. Ketzer, A. Niestroj, S. Schmid, W. Schmid, D. Horváth, J. Eades, and E. Widmann, Phys. Rev. A 52, 4266 (1995).

[12] H.A. Torii, M. Hori, T. Ishikawa, F.E. Maas, R.S. Hayano, N. Morita, M. Kumakura, I. Sugai, B. Ketzer, H. Daniel, F.J. Hartmann, R. Pohl, R. Schmidt, T. von Egidy, D. Horváth, J. Eades, E. Widmann, and T. Yamazaki, Phys. Rev. A 53, R1931 (1996). 
[13] R.S. Hayano, T. Ishikawa, H. Tamura, H.A. Torii, M. Hori, F.E. Maas, N. Morita, M. Kumakura, I. Sugai, F.J. Hartmann, H. Daniel, T. von Egidy, B. Ketzer, R. Pohl, D. Horváth, J. Eades, E. Widmann, and T. Yamazaki, Phys. Rev. A 55, R1 (1997).

[14] T. Yamazaki, E. Widmann, J. Eades, M. Kumakura, N. Morita, H.A. Torii, M. Hori, T. Ishikawa, F.E. Maas, H. Tamura, R.S. Hayano, I. Sugai, Y. Fujita, B. Ketzer, H. Daniel, F.J. Hartmann, M. Hasinoff, R. Pohl, R. Schmidt, T. von Egidy, and D. Horváth, Phys. Rev. A 55, R3295 (1997).

[15] E. Widmann, J. Eades, T. Yamazaki, H.A. Torii, R.S. Hayano, M. Hori, T. Ishikawa, M. Kumakura, N. Morita, I. Sugai, F.J. Hartmann, T. von Egidy, B. Ketzer, C. Maierl, R. Pohl, and D. Horváth, Phys. Lett. B 404, 15 (1997).

[16] T. Yamazaki, B. Ketzer, E. Widmann, J. Eades, H. Daniel, F.J. Hartmann, M. Hasinoff, R. Pohl, R. Schmidt, T. von Egidy, D. Horváth, M. Kumakura, N. Morita, I. Sugai, Y. Fujita, H. A. Torii, M. Hori, T. Ishikawa, F.E. Maas, H. Tamura, and R.S. Hayano, Chem. Phys. Lett. 265, 137 (1997).

[17] B. Ketzer, F.J. Hartmann, T. von Egidy, C. Maierl, R. Pohl, J. Eades, E. Widmann, T. Yamazaki, M. Kumakura, N. Morita, R.S. Hayano, M. Hori, T. Ishikawa, H.A. Torii, I. Sugai, and D. Horváth, Phys. Rev. Lett. 78, 1671 (1997).

[18] B. Ketzer, F.J. Hartmann, T. von Egidy, C. Maierl, R. Pohl, J. Eades, E. Widmann, T. Yamazaki, M. Kumakura, N. Morita, R.S. Hayano, M. Hori, T. Ishikawa, H.A. Torii, I. Sugai, and D. Horváth, J. Chem. Phys. 109, 424 (1998).

[19] M. Hori, H.A. Torii, R.S. Hayano, T. Ishikawa, F.E. Maas, H. Tamura, B. Ketzer, F.J. Hartmann, R. Pohl, C. Maierl, T. von Egidy, M. Kumakura, N. Morita, I. Sugai, D. Horváth, E. Widmann, J. Eades, and T. Yamazaki, Phys. Rev. A 58, 1612 (1998).

[20] T. Yamazaki and K. Ohtsuki, Phys. Rev. A 45, 7782 (1992); K. Ohtsuki (private communication).

[21] I. Shimamura, Phys. Rev. A 46, 3776 (1992); and private communication.

[22] P.T. Greenland and R. Thürwächter, Hyperfine Interact. 76, 355 (1993).

[23] V.I. Korobov, Phys. Rev. A 54, R1749 (1996).
[24] V.I. Korobov and D.D. Bakalov, Phys. Rev. Lett. 79, 3379 (1997).

[25] S. Gerstenkorn, J. Verges, and J. Chevillard, Atlas du Spectre d'Absorption de la Molecule d'Iode (Laboratoire Aimé Cotton CNRS II, Orsay, 1982).

[26] J. Cariou and P. Luc, Atlas du Spectre d'Absorption de la Molecule de Tellure (Laboratoire Aimé Cotton CNRS II, Orsay, 1980).

[27] Cryodata Inc., computer program Helium Thermophysical Properties Software HEPAK, version 3.30, 1992.

[28] E. Yarevsky and N. Elander, Europhys. Lett. 37, 453 (1997).

[29] N. Elander and E. Yarevsky, Phys. Rev. A 56, 1855 (1997); 57, 2256(E) (1998).

[30] S. Andersson, N. Elander, and E. Yarevsky (unpublished).

[31] Y. Kino, M. Kamimura, and H. Kudo, Proceedings of the XVth International Conference on Few-Body Problems in Physics (Groningen, 1997) [Nucl. Phys. A 631, 649c (1998)].

[32] Y. Kino, M. Kamimura, and H. Kudo, in Proceedings of the XVIIth RCNP International Symposium on Innovative Computational Methods in Nuclear Many-Body Problems, Osaka 1997, edited by H. Horiuchi et al. (World Scientific, Singapore, 1998), p. 136; and private communication.

[33] V.I. Korobov, in Proceedings of the International Workshop on Frontier Tests of QED and Physics of the Vacuum, Sandansky, 1998, edited by E. Zavattini, D. Bakalov, and C. Rizzo (Heron Press OOD, Sofia, 1999), and private communication.

[34] G. Gabrielse, D. Phillips, W. Quint, H. Kalinowsky, G. Rouleau, and W. Jhe, Phys. Rev. Lett. 74, 3544 (1995); Particle Data Group, Review of Particle Physics, Eur. Phys. J. C 3, 613 (1998).

[35] R.J. Hughes and B.I. Deutch, Phys. Rev. Lett. 69, 578 (1992).

[36] P. Robertson, T. King, R. Kunselman, J. Miller, R.J. Powers, P.D. Barnes, R.A. Einstein, R.B. Sutton, W.C. Lam, C.R. Cox, M. Eckhause, J.R. Kane, A.M. Rushton, W.F. Vulcan, and R.E. Welsh, Phys. Rev. C 16, 1945 (1977).

[37] Y. Kino, M. Kamimura, and H. Kudo, Proceedings of the International Workshop on Exotic Atoms, Molecules and Muon Catalyzed Fusion, Ascona, 1998 [Hyperfine Interact. (to be published)]. 